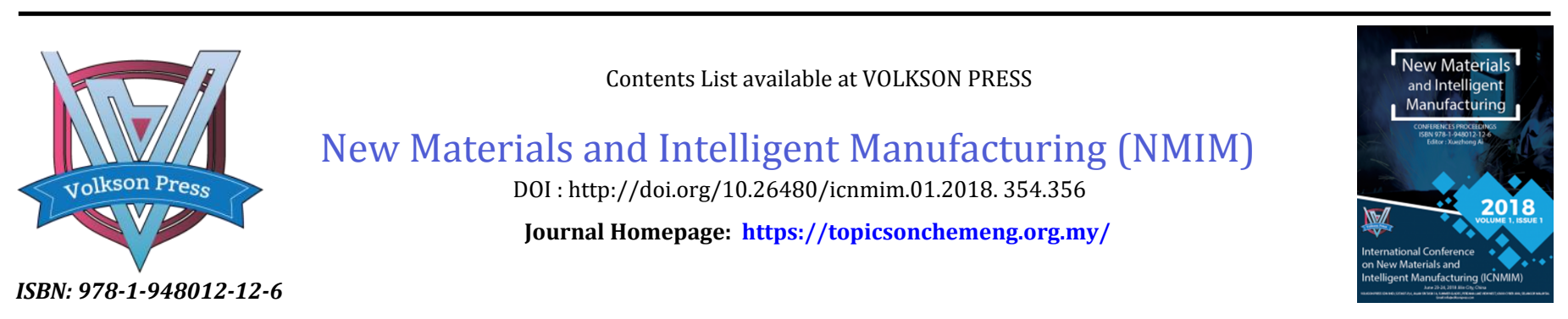

\title{
DEVELOPMENT OF REAL-TIME SIMULATION PLATFORM FOR TANK LEVEL CONTROL SYSTEM BASED ON WINCC
}

\author{
Gao Xingquan'1, Wang Zishuo², Zhao Qiang ${ }^{3}$ \\ ${ }_{1}^{1}$ Professor of Jilin Institute of Chemical Technology, ChengDe Street, Jilin City, China \\ ${ }^{2}$ Control engineering of 1601, Jilin Institute of Chemical Technology, ChengDe Street, Jilin City, China \\ ${ }^{3}$ Control engineering of 1701, Jilin Institute of Chemical Technology, ChengDe Street, Jilin City, China \\ *Corresponding Author Email: 137021135@qq.com
}

This is an open access article distributed ader the Creative Commons Attribution License, which permits unrestricted use, distribution, and reproduction in any medium, provided the original work is properly cited.

\section{ARTICLE DETAILS}

Article History:

Received 26 June 2018

Accepted 2 July 2018

Available online 1 August 2018

\section{ABSTRACT}

This paper uses WinCC configuration software and based on the mathematical model of Coupled-Tank, which writes the program code through C script, solving the tank differential equation by Euler method, and uses the incremental PI algorithm to achieve closed-loop control, and then achieves the purpose of liquid level control. The development of this platform not only enables operators to understand the workflow of the Coupled-Tank, displaying of liquid level changes on real-time, real-time monitoring, and dynamic simulation of liquid level changes of the tank, but also can achieve the effect of online modification of parameters and manual and automatic No disturbance switching in the operating mode.

\section{KEYWORDS}

WinCC, Coupled-Tank, Euler method, No disturbance switching.

\section{INTRODUCTION}

Liquid level control is an important issue in industrial control, and it is widely used in many fields of production. The study of liquid level control not only has a huge impact on the performance and quality of the work, but also relates to personal and property safety of production personnel and production unit [1]. Due to the time-varying and delay characteristics of the liquid level control system, the research process is more complicated. Therefore, in order to facilitate the research, the complex liquid level control system can be abstracted into a Coupled-Tank model [2]. On this basis, in order to reduce the danger and economic loss caused by improper operation or immature technology, it is very important to carry out simulation modeling before working in the water tank. At present, most of the simulations for the Coupled-Tank are MATLAB/Simulink modeling methods. This kind of simulation uses MATLAB's powerful mathematics function, using the mathematics method to calculate the change process of the liquid level of the water tank, and draws the liquid level change curve. However, since all such simulations are mathematical formulas and program codes, the modeling is too abstract, the operator cannot understand the process flow, and cannot modify the parameters in real-time in the reaction process, failing to achieve the monitoring effect [3].

In order to achieve real-time monitoring, real-time display, real-time parameter modification and other purposes, this article has developed a liquid level control system real-time simulation platform based on WinCC configuration software. WinCC is a powerful industrial control software introduced by Siemens, Germany, which integrates data acquisition and monitoring, scripting, configuration and OPC [4]. Using C script can construct the Coupled-Tank mathematical model in WinCC and configure the process flow screen, the operator can simulate the working environment of the tank, run the equipment, and manually adjust the valve opening, It can set the tank liquid level height, the $\mathrm{kp}$ and Ti parameters are modified online and the manual and automatic No disturbance switching effect can be achieved. The development of this platform enables real-time monitoring of the process and liquid level display of the
Coupled-Tank. Operators can easily know the water storage conditions of the entire equipment, greatly reducing the danger of improper operation, and at the same time, it can improve the efficiency of work. This has important implications for the study of liquid level control systems.

\section{THE COUPLED-TANK AND MATHEMATICAL MODEL}

The Coupled-Tank studied in this paper is made up of two tanks connected in series. In the working state, the valve is opened, and the water pump feeds the water in the tank to the upper tank. Due to the gravity and pressure of the water in the water tank, the water flows from the outlet at the bottom of the upper water tank into the lower water tank, and then it flows back to the reservoir from the bottom outlet hole of the lower water tank to form a closed-loop. By changing the valve opening, the inlet flow rate can be controlled to achieve the purpose of the lower tank level control. Figure 1 shows a schematic diagram of the Coupled-Tank [5].

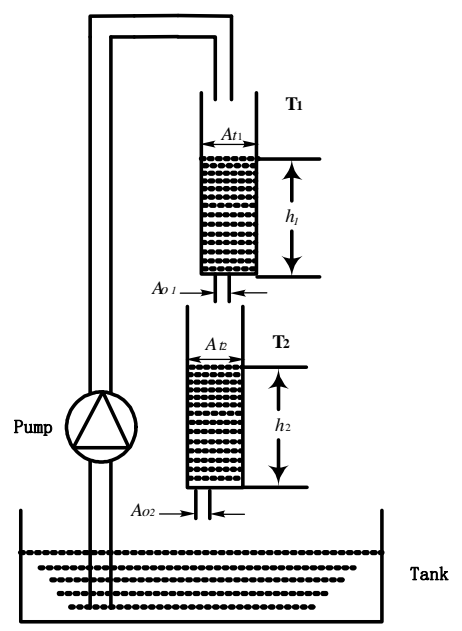

Figure 1: Coupled-Tank structure 
According to the material balance, the Coupled-Tank system can be described by the following nonlinear differential equations:

$$
\begin{aligned}
& A_{t 1} \frac{d h_{1}}{d t} \quad 22 k_{p} o p / 100-a z_{1} A_{o 1} \sqrt{2 g h_{1}} \\
& A_{t 2} \frac{d h_{2}}{d t}=a z_{1} A_{o 1} \sqrt{2 g h_{1}}-a z_{2} A_{o 2} \sqrt{2 g h_{2}}
\end{aligned}
$$

Where $h_{i}$ is $i$ the level of the tank, $A_{t 1}=A_{t 2}=15.5179 \mathrm{~cm}^{2}$ is the crosssectional area of the two tanks, $A_{01}=0.1781 \mathrm{~cm}^{2}, \quad A_{o 2}=0.1781 \mathrm{~cm}^{2}$ is the cross-sectional area of the connecting pipe of the tank, $g=981 \mathrm{~cm} / \mathrm{s}^{2}$ is the gravitational acceleration, $K_{p}=3.3$ is the flow coefficient of the pump, and op is the opening of the regulating valve.

\section{OVERALL FRAMEWORK OF THE SIMULATION PLATFORM}

The most important function of the WinCC monitoring system is the controllability and visualization of the process. It is necessary to build the screen to be able to restore the working status of the equipment in order to achieve the monitoring purpose of change. In this simulation platform, four areas that the process flow, parameter display, variable setting, and real-time change trend of the Coupled-Tank are included. In the process flow screen, the operator can intuitively recognize the situation on the site according to the configuration screen, and the operator also can simulate the actual Coupled-Tank working status, start the equipment, and select manual control or automatic control. In the parameter display and variable setting area, in order to facilitate the operator to change the variable value and can modify the liquid level height setting value and the PI parameter online, all variables should be displayed in the form of numbers and bar graphs. In the real-time trend area, use the WinCC online Trend Control in the Graphics Designer to display the level of the CoupledTank level and the valve opening. Coupled-Tank real-time simulation platform is shown in Figure 2.

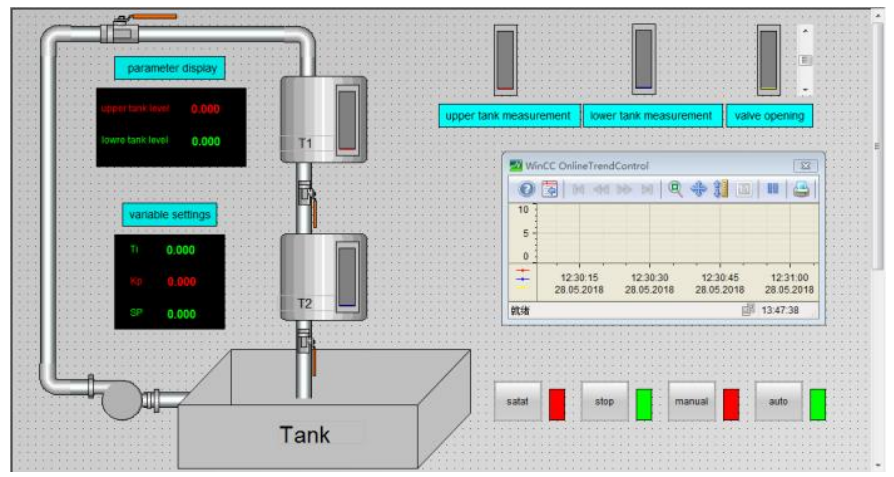

Figure 2: Coupled-Tank real-time simulation platform

\section{SIMULATION AND IMPLEMENTATION OF DYNAMIC CHARACTERISTICS OF COUPLED-TANK}

A key and key issue of this simulation platform is how to describe the realtime changes of liquid level.

\subsection{Monitoring Interface Configuration}

WinCC uses the changes in the physical properties (color, position, size, etc.) of the object to reflect the state of the hardware system's running process, or to control the system's running process through the buttons in the operation screen. The so-called dynamic simulation is to connect a variable with a certain physical quantity attribute of the object, and the value of the variable is automatically assigned to the object. When the value of the variable changes, the attribute of the object also changes accordingly. In order to achieve dynamic simulation of the system, the internal variables should first be established in the variable management, in which the floating-point 32-bit IEEE 745 variable contains the upper tank level height is $h 1$, the lower tank level height is $h 2$, the valve opening is op, and the liquid level setting is sp, proportional control is kp, integral is $\mathrm{Ti}$, binary variables, Including start, stop, manual control, automatic control. Secondly, it connect the tank variable in the configuration screen, and use the bar graph to display the ratio of the liquid level height to the tank height. At the valve opening, with add the scroll bar operation keys to facilitate the operator to perform manual control. The upper limit is set to $100 \%$, the change of the liquid level value can be clearly observed after connecting the upper and lower tank water level in the parameter display area. In order to make the user intuitively observe the running status of the device, We should add a button color change display at the beginning, right-click graphic properties, select "Effect", and change the "Global Color Scheme" to No, so that when the action changes, the graphic shows different colors depending on the action. Finally, in the control panel, stand-alone" ActiveX controls", select "WinCC online Trend Control" to add the trend curve control, right-open "WinCC online Trend Control" properties dialog box. In the trend, it connects the upper and lower water tanks, valve opening online variables, and set the value axis scale to complete the entire dynamic simulation build [6].

\subsection{Model Solving}

After the screen configuration is completed and the variables are connected, the mathematical differential equations of the Coupled-Tank are solved to obtain the real-time variation of the two tank water level. In the initial state of the Coupled-Tank, the heights of the two tanks are all 0 $\mathrm{cm}$. For the differential equation of a given starting point, Euler's method can be used to solve the problem. According to Euler's method, the tank differential equation is rewritten as the difference equation, where Ts is the tank level change period, every interval Ts seconds, the tank level is iterated once, successively replaced, finally found the height change of the tank level. The dual tank differential equation is shown below.

$$
\left\{\begin{array}{l}
h_{1}(1)=h_{1}(0)+T s\left(\frac{22 k_{p} o p / 100}{A_{t 1}}-\frac{a z_{1} A_{o 1} \sqrt{2 g h_{1}(0)}}{A_{t 1}}\right) \\
h_{2}(1)=h()+T s\left(\frac{a z_{1} A_{1} \sqrt{2 g h_{1}(0)}}{A_{t 2}}-\frac{a z_{2} A_{o 2} \sqrt{2 g h_{2}(0)}}{A_{t 2}}\right)
\end{array}\right.
$$

\subsection{C script implementation}

C script reads the system internal variable, assigns the variable value to the function to carry on the computation, then obtains the solution, according to the difference equation of the Coupled-Tank, first in the blank place in the graph editor, right key selects the attribute, then selects the $\mathrm{C}$ action in the display to program code, enters "static float", sets the initial value of parameters such as tank level. Secondly, under manual control, it click on the internal function, "Get Tag Bit" to get the starting and manual control variables, and write the differential equation of the Coupled-Tank through C language. Finally, through the "Set Tag Float" output operation to get the upper and lower tank level height and valve opening, the same way to set automatic control. But the automatic control needs to set the liquid level ,set height sp, and the proportional coefficient kp, integral coefficient Ti. After the PI operation, the height of the liquid level is output, and in order to ensure the authenticity and effectiveness of the system operation, the maximum valve opening is limited to $100 \%$. Since the water level, valve opening, and other parameter data types belong to the floatingpoint 32-bit IEEE 745 , the calculation cycle of the variable is $12.5 \mathrm{~ms}$, and the maximum one cycle runtime is $87.5 \mathrm{~ms}$. In order to ensure real-time platform development, the time-varying period in the script trigger is 250ms [7].

\section{MANUAL CONTROL AND AUTOMATIC CONTROL}

\subsection{Manual Control}

Activate the system, run the $\mathrm{C}$ script, click the start button, select the manual control, adjust the valve opening to $50 \%$, observe the tank level change in real-time in the "WinCC online Trend Control" control, and the valve opening will not change over time, the flow of the inlet is certain, the two tanks are constantly rising in liquid level, and they finally stabilize at a certain height. The liquid level change curve is shown in Figure 3. 


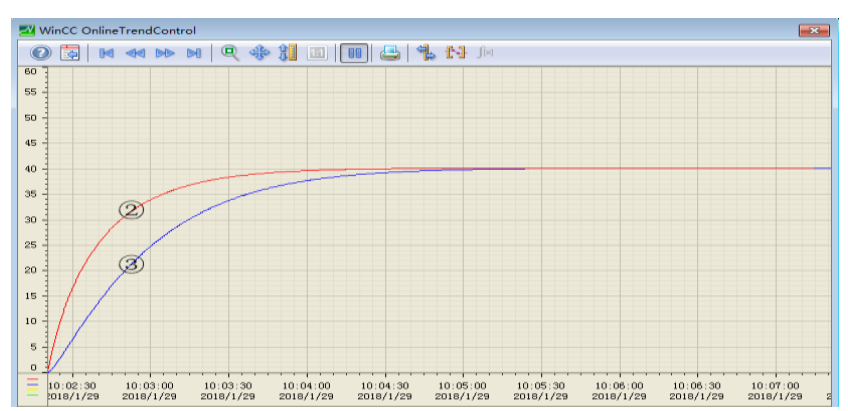

Figure 3: Change of the liquid level of the Coupled-Tank in the manual state

In the figure, (2) is the change curve of the level of the upper tank, and (3) is the change curve of the level of the tank.

\subsection{Automatic Control}

In the automatic control state, the choice of control method is of utmost importance. In order to save computer memory, calculate the time used, and achieve manual and automatic no disturbance switching effect, the resulting system oscillation is small and the platform achieves the effect of rapid operation. The Coupled-Tank real-time simulation platform adopts incremental PI control method and the PI control formula is as follows.

$$
u_{i}=u_{i-1}+K_{P}\left(1+\frac{T}{T_{I}}\right) e_{i}-K_{P} e_{i-1}
$$

In the platform, when the system is switched to the automatic working mode, the tank level height is set, and the actual level of the lower tank is compared with the set value. After using the obtained difference to do the PI calculation, updating the parameters and adjusting the valve opening to achieve the tank level control. Where $u_{i}$ is the voltage of the pump that provides the flow to the upper tank, $e_{i}$ is the difference between the tank's level at the current time and the lower tank's level setting, and $T$ is the tank's simulation cycle.

Run the system in the automatic state. In the variable setting area, set the tank level to $20 \mathrm{~cm}$, kp is set to $0.5, \mathrm{Ti}$ is set to 9 , and the system operation result is shown in Figure 4.

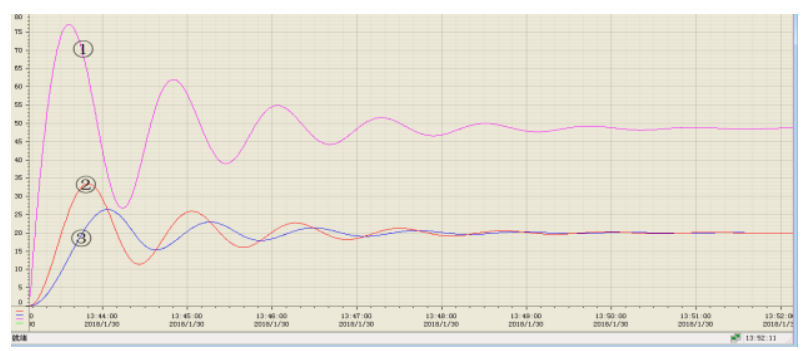

Figure 4: Liquid level change curve of Coupled-Tank in automatic state

In the figure, (1) is the valve opening change curve, (2) is the upper tank level change curve, and (3) is the lower tank level change curve.

\subsection{Manual and automatic no disturbance switching}

In actual operation, when the manual and automatic switch to each other, the height of the liquid level does not change. In order to highly restore the working status of the Coupled-Tank, a manual and automatic no disturbance switching method is set on the platform. In the process of system operation, manual and automatic no disturbance switching are performed. In automatic control, the set value of manually controlled valve opening can be continuously updated. When it is switched to automatic, the manually controlled valve opening is used as the initial value of the operation during automatic control. In the same way, in order to ensure that the valve opening and the pump do not have large abrupt changes, when the valve is switched to manual control, the opening of the valve must be maintained at the final value of the automatic control of the opening of the valve. In the liquid level control of the tank, manual $\rightarrow$ automatic, automatic $\rightarrow$ manual process curve, as shown in Figure 5

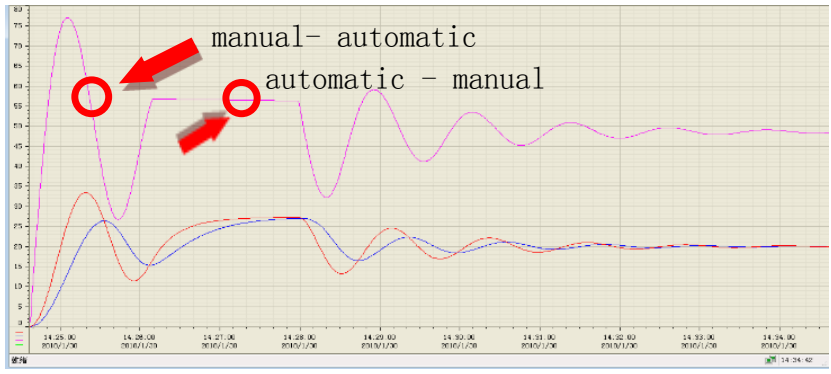

Figure 5: Level curve changes of manual and automatic no disturbance switching

\section{ALARM MESSAGE}

The news is a very important part of WinCC, used to monitor the abnormal situation of the working state, the design of this platform has configured the alarm for the height level of the tank and the valve opening. Using the "WinCC Alarm Control" control to display an alarm message when the liquid level is above or below the liquid level maximum, operators can quickly find and locate, and resolve faults and errors with the help of monitoring screens or sounds, thereby reducing downtime and production accidents and ensuring that real-time simulation platforms can achieve true simulation results.

\section{CONCLUSION}

In this paper, the $\mathrm{C}$ script system of the configuration software WinCC is used to simulate the real-time simulation of the level change of the Coupled-tank. Not only can the process control of the control system be monitored, but also it can reflect the dynamic change of tank level, the process of changing the liquid level is clearly and intuitively displayed, and the liquid level control is finally achieved. Experimental results show: In the WinCC configuration animation, the change of the system curve can be changed by adjusting the valve opening in the manual state, setting the liquid level height in the automatic state or modifying the parameters in the online control system, and the manual state or the automatic state is switched to each other, the system does not have a sudden change and achieve a good stable operation. The development of this platform is of great significance to the operator's familiarity with the actual production process, and at the same time provides great convenience for experiments and learning.

\section{REFERENCES}

[1] Dang, Y., Wang, H.B., Liu, T.W., Yang, S.R., Zhao, Y.J. 2013. The Design of Human-Machine Interface for Rudder Control System Monitoring Platform Based on WinCC [J]. Applied Mechanics and Materials, 2560 (347).

[2] Zheng, M.G., Zhu, H.X. 2012. Design of Tower Crane Intelligent Monitoring Management System Based on PLC and WinCC [J]. Applied Mechanics and Materials, 1867 (184).

[3] Shao, D., Liu, H.T., Chen, H.Y. 2013. Furnace Data Management System Based on WinCC User Archive [J]. Advanced Materials Research, 2450 (712).

[4] Zhao, M., Ren, C.Y., Lei, D.L., Qi, F.L 2013. The Study on Liquid Level Control System Based on PLC and WinCC [J]. Applied Mechanics and Materials, 2491 (336).

[5] Wang, D.T., Yao, D.D. 2012. Design of Photovoltaic Generation Monitoring System Based on WinCC [J]. Advanced Materials Research, 1518 (383).

[6] Sen, S.C., Kasim, S., Fudzee, M.F.M., Abdullah, R., Atan, R. 2017. Random Walk from Different Perspective. Acta Electronica Malaysia, 1 (2), 26-27.

[7] Li, B., Li, Z. 2018. The Implement of Wireless Responder System Based on Radio Frequency Technology. Acta Electronica Malaysia, 2 (1) 15-17. 